\title{
ERRATUM \\ New scaling laws for turbulent Poiseuille flow with wall transpiration - ERRATUM
}

\author{
V. Avsarkisov, M. Oberlack and S. Hoyas \\ doi:10.1017/jfm.2014.98, Published by Cambridge University Press, \\ 28 March 2014
}

Key words: turbulence simulation, turbulence theory, erratum

The original version of Avsarkisov, Oberlack \& Hoyas (2014) was published with the incorrect affiliation for S. Hoyas. The correct affiliation is:

CMT Motores Térmicos, Universitat Politècnica de València, València, Spain The error has been rectified in the online PDF and HTML copies.

\section{REFERENCE}

Avsarkisov, V., Oberlack, M. \& Hoyas, S. 2014 New scaling laws for turbulent Poiseuille flow with wall transpiration. J. Fluid Mech. 746, 99-122. 